\title{
Materials development for new high heat-flux component mock-ups for DEMO
}

\author{
J.W. Coenen ${ }^{\mathrm{a}}$, Y.Mao ${ }^{\mathrm{a}}$, S.Sistla ${ }^{\mathrm{c}}$, A.v.Müller ${ }^{\mathrm{b}, \mathrm{d}}$, G.Pintsuk $^{\mathrm{a}}$, M.Wirtz ${ }^{\mathrm{a}}$, J.Riesch ${ }^{\mathrm{b}}$, T.Hoeschen ${ }^{\mathrm{b}}$, A.Terra ${ }^{\mathrm{a}}$, J-H.You ${ }^{\mathrm{b}}$, H.Greuner $^{\mathrm{b}}$,

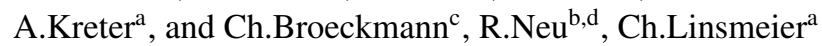 \\ ${ }^{a}$ Forschungszentrum Jülich GmbH, Institut fuer Energie und Klimaforschung, Partner of the Trilateral Euregio Cluster (TEC), 52425 Juelich, Germany \\ ${ }^{b}$ Max-Planck-Institut für Plasmaphysik, 85748 Garching, Germany \\ ${ }^{c}$ Lehrstuhl für Werkstoffanwendungen im Maschinenbau, RWTH Aachen, 52062 Aachen Germany \\ ${ }^{d}$ Technische Universität München, Boltzmannstrasse 15, 85748 Garching, Germany
}

\begin{abstract}
Material issues pose a significant challenge for future fusion reactors like DEMO. When using materials in a fusion environment a highly integrated approach is required. Damage resilience, power exhaust, as well as oxidation resistance during accidental air ingress are driving issues when deciding for new materials. Neutron induced effects e.g. transmutation adding to embrittlement are crucial to material performance. Here advanced materials such as tungsten fiber-reinforced tungsten $W_{f} / W$ and fiber-reinforced copper $W_{f} / C u$ composites could allow the step towards a fusion reactor. Recent developments in the area $W_{f} / W$ mark a possible path towards a component mock-up early enough for utilisation in DEMO. High heat-flux tests show that having short fibres at the exposed surface leads to their selective erosion and melting. Initial tests in the linear plasma device PSI-2 confirm this behaviour.
\end{abstract}

Keywords:

\section{Introduction}

Tungsten (W) is currently the main candidate as plasma facing material for the highly loaded divertor components of any future fusion reactor as it is resilient against erosion by impinging plasma particles and neutrals, has the highest melting point of any metal, and shows rather benign activation behavior under neutron irradiation. In addition, low tritium retention is a beneficial property. In recent years many of studies have tackled the issue to qualify current materials with respect to these issues for ITER $[1,2,3,4,5,6,7,8,9,10,11,12,3]$ and beyond. For a DEMO type device, or a future fusion power plant the limits are more stringent. It is assumed that the boundary conditions $[2,13]$ to be fulfilled for the materials are in many cases above the technical feasibility limits as they are set out today $[11,12]$. Efforts to establish new advanced plasmafacing material-options are moving forward (see [12, 14, 9], and references therein) focussing on crack resilient materials with low activation, minimal tritium uptake, long lifetime and low erosion. Many advanced materials base their improved properties on the use of a composite approach. One concept is based on the incorporation of fibres where energy dissipating mechanisms, like ductile deformation of fibres, fibre pull-out, and crack bridging and deflection are facilitated [15, 16, 17].

An issue not tackled in this contribution is the formation of radioactive and highly volatile $\mathrm{W}$-oxide $\left(\mathrm{WO}_{3}\right)$ compounds during accidental air ingress. To suppress the release of $\mathrm{W}$ oxides W-based self-passivating alloys can be incorporated into the composite approach [18, 19, 20, 21, 22, 23, 24, 25, 26, 27].

The operational gap between materials for cooling structures e.g. $\mathrm{Cu}$ alloys, and the plasma-facing materials needs to be bridged $[28,7]$. The recommended operating temperature window for $\mathrm{Cu}, \mathrm{CuCrZr}$ is limited from approximately $453 \mathrm{~K}$ to $573 \mathrm{~K}$ due to embrittlement under neutron irradiation at lower and loss of strength at elevated temperatures [28, 29]. Together $\mathrm{W} / \mathrm{Cu}$ and $W_{f} / W$ composites can bridge the temperature gap between the upper bound for strength of $\mathrm{Cu}$ alloys and the DBTT of $\mathrm{W}$ of up to $800{ }^{\circ} \mathrm{C}$ [30] and embrittlement by recrystallisation and extensive grain grotwh above $1250^{\circ} \mathrm{C}[31,28]$.

In this contribution the focus lies on the methods employed to develop first mock-up systems of the materials for high heatflux qualification, initial results and further plans to drive component development including $W_{f} / C u$ [7] and $W_{f} / W$ with the focus on the tungsten composite qualification.

\section{Tungsten-Fibre Reinforced Copper}

Tungsten (W) fibre-reinforced copper $(\mathrm{Cu})\left(W_{f} / \mathrm{Cu}\right)$ is currently regarded as an advanced heat sink material for highly heat loaded plasma-facing components (PFCs) of future thermonuclear fusion devices $[2,7]$. This is mainly due to the following reasons: $\mathrm{W}-\mathrm{Cu}$ composite materials do in principle exhibit a high thermal conductivity due to a coherent $\mathrm{Cu}$ or $\mathrm{Cu}$ alloy matrix as well as improved strength properties at elevated temperatures. This is required to overcome the above mentioned issue of material operating temperature gap between the heat sink and the plasma-facing material within a PFC. The preferred heat sink material currently used for PFC applications is the precipitation hardened $\mathrm{CuCrZr}$ Alloy [32, 33, 34, 35]. $W_{f} / C u$ composite materials are practically relevant as the constituent materials for such a composite are readily available and the composite can be produced straightforwardly by means of liquid $\mathrm{Cu}$ melt infiltration of open porous $\mathrm{W}$ fibre preforms. The preferred reinforcing fibres for $W_{f} / C u$ are commercially 
available drawn potassium $(\mathrm{K})$ doped $\mathrm{W}$ fibres which are the same reinforcements as also used within the $W_{f} / W$ materials. As $W_{f} / C u$ is a material that is of direct interest with regard to PFC heat sink application the fabrication of cylindrical preforms suitable for further processing to heat sink pipes for application in actively cooled components is evident. Hence, the possibility to produce such preforms out of $\mathrm{W}$ fibres by means of braiding has been demonstrated [36]. In figure 1, a multilayered circular braiding is shown. The magnified SEM image of the braiding illustrates the arrangement of the $\mathrm{W}$ fibres within the preform which have a nominal diameter of $50 \mu \mathrm{m}$.

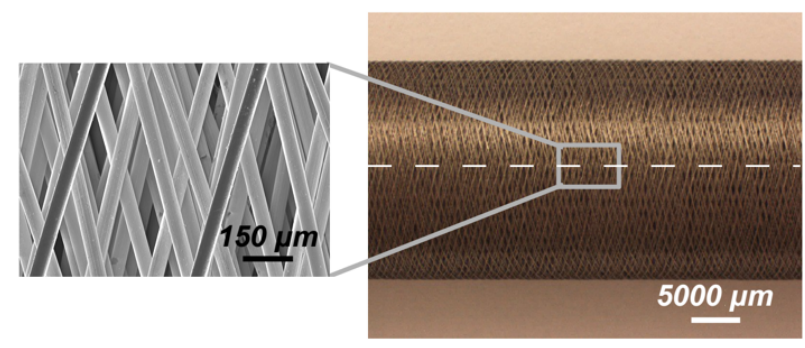

Figure 1: Cylindrical multi-layered braiding made out of continuous W fibres with a nominal diameter of $50 \mu \mathrm{m}$

In figure 2 [7], the result of a $\mathrm{Cu}$ melt infiltration of a braided preform as illustrated in figure 1 is shown. It can be seen that the $W_{f} / C u$ composite material exhibits a microstructure without porosity or voids - as presented in [7, 37].
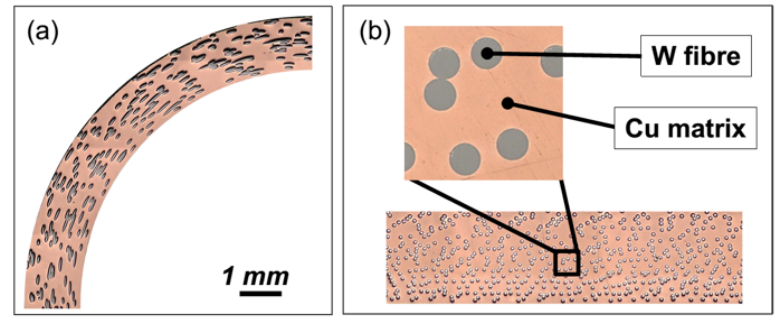

Figure 2: (a) Transverse as well as (b) axial microsection of a Wf-Cu heat sink pipe produced by means of liquid $\mathrm{Cu}$ melt infiltration of a preform as illustrated in figure 1 [7]

Component Mock-ups using $W_{f} / C u$ have already been produced and are undergoing testing [2].

\section{Tungsten-Fibre Reinforced Tungsten}

To overcome the brittleness issues when using $\mathrm{W}$, a $\mathrm{W}$ fibre enhanced $\mathrm{W}$ composite material $\left(W_{f} / W\right)$, incorporating extrinsic toughening mechanisms can be used.

Various methods of building and constructing $W_{f} / W$ composites, either via Chemical Vapor Deposition (CVD) [38, 39] or powder metallurgical (PM) processes $[40,41]$ are available. Based on the work presented here and previous work [40, 42, $43,44,45,36,46]$, the basic proof of principle for CVD \& $\mathrm{PM}-W_{f} / W$ has been achieved. One of the crucial issues is to maintain as much of the properties of the constituents even after exposing the material to the production cycle and the fusion environment allowing for optimal extrinsic toughening and pseudo-ductile behaviour. Here mainly the weak interface and the strength of the fibre is important. Yttria is an ideal candidate as the interface material for the $W_{f} / W$ composite due to its several advanced properties: good thermal and chemical stability, high mechanical strength and hardness [14, 47] as well as low neutron activation.

With respect to the constituent properties it can be expected that when using doped $\mathrm{W}$-wires they will retain their ductility even at elevated temperatures (above $1500 \mathrm{~K}$ ) [42] and all mechanisms necessary for pseudo-ductility will be enabled [14, 43, 45]. Properties of the fibres can however be degraded by various circumstances e.g. by impurities during fabrication [48, 49], high-temperatures or neutron irradiation during operation $[30,50]$. For the purpose of this paper material produced via

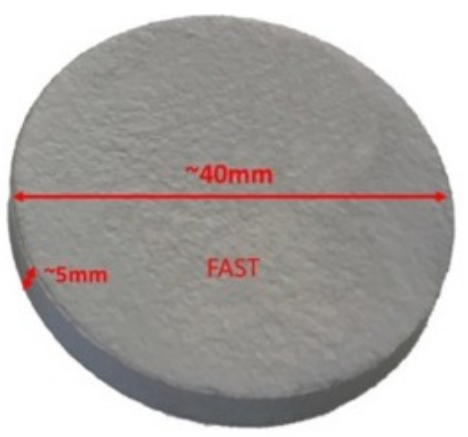

Figure 3: $W_{f} / W$ Sample after FAST

(Field Assisted Sintering Technology) FAST as described in $[45,46,48]$ is used. The typical sample size produced is a $40 \mathrm{~mm}$ disc as depicted in figure 3 . For the samples produced here $1.5 \mathrm{~mm}$ long and $0.15 \mathrm{~mm}$ thick tungsten fibres have been used. Two cases of interface are considered and used in two experiments, respectively. No-interface and an yttria interface between fibers and matrix. The density of the samples is $~ 94 \%$ [46]. The interface is applied bvia a PVD process as described in $[47,46]$ Figure 4 shows the typical microstructure of a PM-

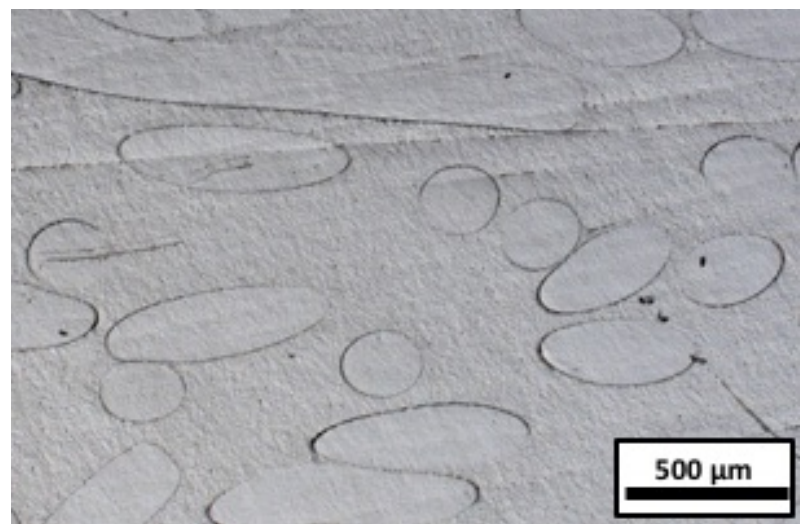

Figure 4: Overview Microstructure after sintering. 
$W_{f} / W$ sample after cutting and polishing. The typical random distribution of the fibers is visible and exposed. Typically $W_{f} / W$ shows stable crack propagation already at RT as well as enhanced fracture toughness well above conventional as sintered W. $W_{f} / W$ samples in the studies produced above show roughly a four times increase in fracture toughness over the pure $\mathrm{W}$ reference.

\section{High Heat Flux Tests Samples}

For the qualification of new materials for fusion one typical test is high heat-flux exposure under controlled condition of both an electron beam gun such as JUDITH-1 [51] as well as joint or subsequent loading with plasma and laser in e.g. a linear plasma device such as PSI-2 [52]. As part of the materials program within the EUROfusion consortium neutron irradiation tests of new materials incl. $W_{f} / W$ are being performed.

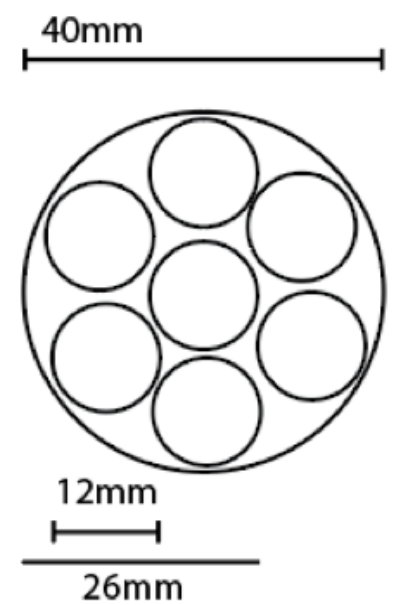

Figure 5: Samples dimensions cut from the original 40mm disc

For these neutron irradiation studies dedicated samples have been produced to later be tested in both JUDITH-1 [51] and also the linear plasma device JULE-PSI [53]. These samples were cut from the $40 \mathrm{~mm}$ discs of PM short fibre $W_{f} / W$ as depicted in fig. 5. For details fo the production process refer to $[48,46,14,45]$. The samples are cut into $12 \mathrm{~mm}$ diameter discs. They contain $30 \%$ volume fraction of yttria coated $1.5 \mathrm{~mm}$ long and $0.15 \mathrm{~mm}$ thick tungsten fibers. The samples dicussed and exposed for the presented work ( in JUDITH-1) are the nonirradiated reference samples for later comparison with tests of irradiated samples.

For exposures in PSI-2 [52] different types of sample are used with respect to geometry. Fig. 6 gives a representation of the samples cut from the raw material and exposed in PSI-2. These samples similarly contain $30 \%$ volume fraction of fibers, in this case without yttria coating and are cut from a similar disc of PM $W_{f} / W$.

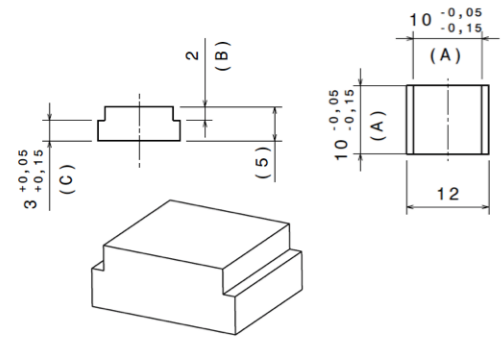

Figure 6: Sample geometry used for exposure in PSI-2[52]

\section{High Heat Flux \& Plasma Tests}

For the initial high heat flux tests a limited number of samples was used to establish the behaviour of $W_{f} / W$ under electron beam loading. For details of the devices used please refer to $[51,8]$. Samples were mounted without active cooling and pre-heated to $1000^{\circ} \mathrm{C}$. The samples were all exposed to 1000 heat pulses and to different values of impinging heatflux. 1: $0.19 \mathrm{GW} / \mathrm{m}^{2}$ for $1 \mathrm{~ms} ; 2: 0.28 \mathrm{GW} / \mathrm{m}^{2}$ for $1 \mathrm{~ms} ; 3: 0.38 \mathrm{GW} / \mathrm{m}^{2}$ for $1 \mathrm{~ms}$; $4: 1.13 \mathrm{GW} / \mathrm{m}^{2}$ for $5 \mathrm{~ms}$. This represents heat flux factors $\left(F_{H F}\left[M W /\left(m^{2} s^{0.5}\right)\right]\right)$ [3] of $6,9,12$ and 80 respectively, describe in basic terms the absolute temperature rise with respect to each other [54]. The samples are thus exposed to heat fluxes around and significantly above the melting threshold for pure tungsten (see below).

Figure 7 displays the samples after exposure, the top row shows the exposed surface, while the bottom row is a crosssection through the material. It can be observed that with increasing heat flux more and more damage is visible. For sample \#1 the onset of a crack network is visible with cracks mostly confined to the surface. For sample \#2 with increasing heat flux, the crack network increases and in the section of the sample after exposure delaminated fibres are visible, which leads to initial melting of the top surface fibres due to loss of thermal contact. This behaviour increases in sample \#3 where a multitude of fibers are bent, melted or debonded. The cracks throughout the matrix are even more visible.

"For pure tungsten it is known that single or few intense thermal shocks can lead to cracking of the surface with little to no fatigue effects involved. The thresholds above which W (99.97 wt.\%, forged) starts to crack after 100 transient heat loads of $1 \mathrm{~ms}$ at room temperature are typically between 0.19 $0.38 \mathrm{MJ} / \mathrm{m}^{2}\left(F_{H F}=6-12 \mathrm{MW} /\left(\mathrm{m}^{2} \mathrm{~s}^{0.5}\right)\right)$ but strongly depend on microstructure and composition" [3].

For sample \#4 strong melting of the top surface of the sample is observed. Cracks propagate into the top layer and beyond where melting has opened up the material. It should be stated that melting of the bulk is definitely beyond the operational point envisioned for a component. As mentioned above the test in PSI-2 were performed with different samples not utilising an yttria interface. The samples were loaded for 1000 times at $0.38 \mathrm{GW} / \mathrm{m}^{2}$ for $1 \mathrm{~ms}$ while being loaded in PSI-2 with a D-He plasma for 2520 seconds. The overall erosion rate is 

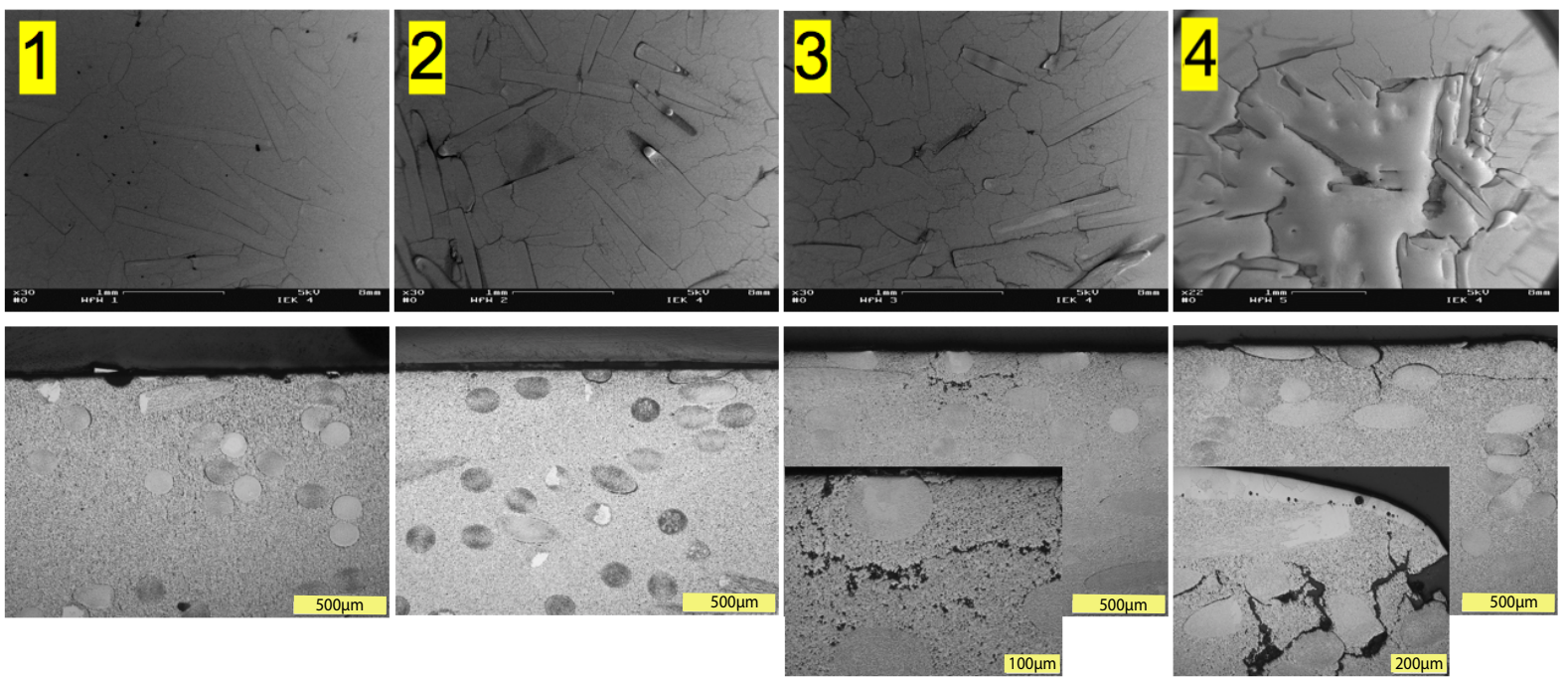

Figure 7: TOP: Exposed surface after high heat-flux exposure; BOTTOM: Metallurgical section though the exposed sample. $1: 0.19 \mathrm{GW} / \mathrm{m}^{2}$ for $1 \mathrm{~ms} ; 2: 0.28$ $\mathrm{GW} / \mathrm{m}^{2}$ for $1 \mathrm{~ms}$; 3: $0.38 \mathrm{GW} / \mathrm{m}^{2}$ for $1 \mathrm{~ms}$; $4: 1.13 \mathrm{GW} / \mathrm{m}^{2}$ for $5 \mathrm{~ms}$

$1.7 \mu \mathrm{g} / \mathrm{s}$ after both plasma and laser exposure. The roughness increases from $R_{a}=0.079$ to $R_{a}=0.154$.

The heat load applied via laser is very similar to the above discussed samples \#3 exposed in JUDITH-1 despite small variations in the shape of the loaded area ( $4 \times 4 \mathrm{~mm}$ in JUDITH- 1 vs $3 \mathrm{~mm}$ diameter for the laser in PSI-2). The main difference here is that in contrast to the sample exposed in JUDITH-1 no interface is used between fibers and matrix. This becomes clearly visible in the behaviour of the individual fibers. Where as in the JUDITH-1 exposures the fibres exposed in the top layer of the sample are starting to melt and bend this does not occur in the PSI-2 exposed samples. For the PSI-2 samples only a clear crack network is visible similar to the cracks observed above in the matrix material. The cracks do not penetrate into the fibres, hence hinting at crack deflection around the embedded fibers.

The bending and melting visible in figure 7 can be attributed to the weak and thermally insulating interface present on the fibres. As this is not included in the samples exposed to PSI-2 and shown in figure 8 the conclusion is that exposing the fibres to the top surface will have to be considered when designing a component as already discussed in [55]. The above given results show that for the material used here no significant difference in cracking behaviour under transient heat-loads can be found when considering only the matrix. We will discuss below what this means for utilising $W_{f} / W$ as divertor component in future fusion devices. One issue that definitely warrants attention as well is the preferential melting or bending of the fibers integrated into the top surface.

\section{Preparing for a new Divertor Component}

In [55] inital diussions were presented on what structure a potential DEMO mock-up incorporating new materials should have. "By introducing either alloys or composite structures one does change significantly the behavior of the components with respect to plasma-wall interaction. First and foremost the
Before
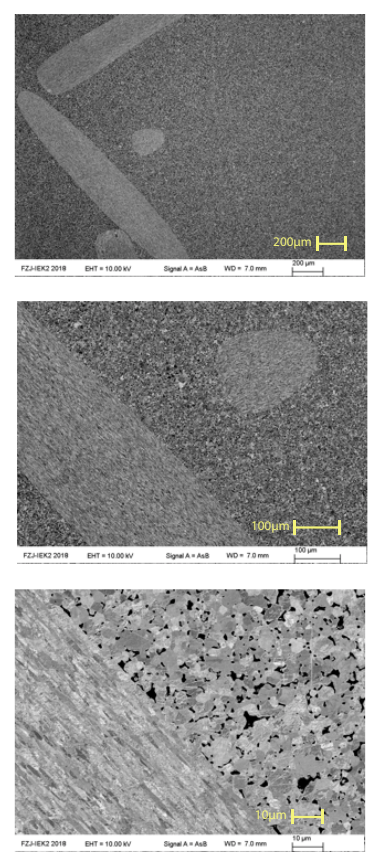

After
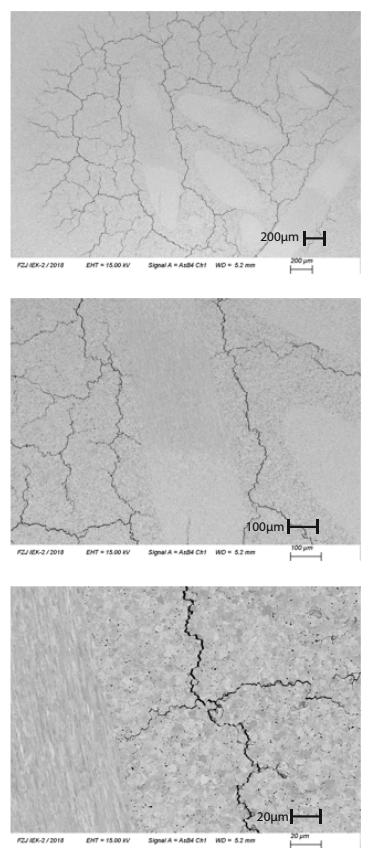

Figure 8: Surfaces of the $W_{f} / W$ sample exposed in PSI-2 before and after laser and plasma exposure. (1000 pulses at $0.38 \mathrm{GW} / \mathrm{m}^{2}$ for $1 \mathrm{~ms}$ ) 
changes are linked to erosion behavior and lifetime concerns and the retention and interaction with plasma species like hydrogen and helium. A typical model component consists of a tungsten fiber reinforced tungsten $\left(W_{f} / W\right)$ [42], smart alloy $[18,56,57,58,59]$ with interfaces based on oxide ceramics, a copper based cooling tube and integrated permeation barrier layers [60] (Fig. 9)". Size arguments on the component and there dimensions can be found in [61]. Based on [55] and [7]

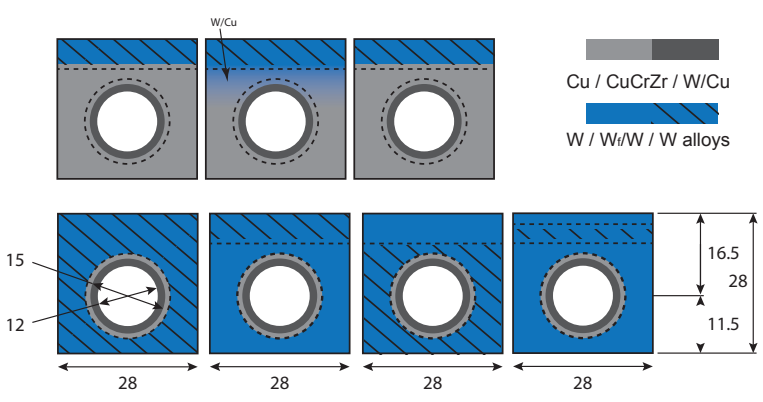

Figure 9: Based on the current designs chosen for ITER and DEMO the monoblock or flat-tile design are favored [Flat Tile (Top) Bulk Material (Bottom)]. Introducing the advanced materials and composites (e.g. $W_{f} / W W_{f} / C u$ ) can however be done in various locations - Dashed lines indicate locations of material interfaces and potential locations of permeation barriers. Dimensions are given in $\mathrm{mm}$

and the above made observation further tests can be planned and a component design suggested.

From the results presented here it becomes more clear that an armour layer might be required to protect the top layer of $W_{f} / W$. Here the interface and the thermal contact of the fibers in the material is crucial.

Fig. 9 displays multiple options for a component either utilising $W_{f} / W$ in bulk or including a tungsten armour as the topmost layer. In all cases it seems reasonable to include a $W_{f} / C u$ tube to take advantage of the better high temperature properties and thus mitigating potential issues of an existing operational gap in the interfaces regarding thermal and mechanical properties. Both mono-block like structures but also flat-tile designs could be envisioned. The later basically combines a tungsten armour on top of a massive copper based cooling structure.
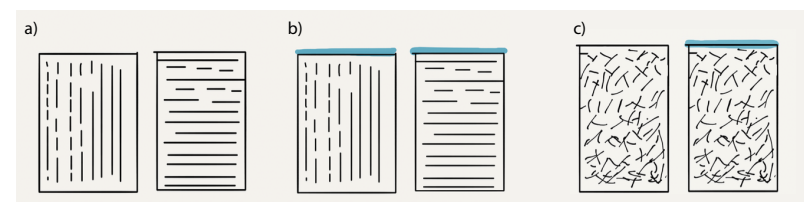

Figure 10: Sketch of potential test samples used in GLADIS [62] with and w/o tungsten armour layer on top. a) both typical long fibre $W_{f} / W$ orientations without additional armour, b) with additional armour layer, a) short fibre $W_{f} / W$ with and w/o armour layer

As a next step tests on at least 4 classes of $W_{f} / W$ are thus envisioned. Figure 10 shows CVD $W_{f} / W$ including long fibers either horizontally or vertically to the surface, as well as similar material type for short fibre $W_{f} / W$. These materials are cur- rently produced to be tested on an actively cooled copper substructure in the neutral beam high heat flux test facility GLADIS [62] within 2019. Here the focus is both on the thermo-mechanical stability of the $W_{f}$ matrix interface, potential cracking and stable crack growth during loading and establishing if and how a potential armour layer will work to protect the top fibre layer. The influence of hydrogen loading at high heat fluxes will also be studied. As an armour layer pure tungsten either from a CVD or PM process is envisioned as well as the potential application of a new armour material - micro-structured tungsten [63]

\section{Conclusion and Outlook}

In $[14,43,45,46,48,44]$ it was established that stable crack growth and especially the resilience against crack growth is the overarching aim when producing $W_{f} / W$. From the results presented here it is clear that cracking of the top layer might not be able to be prevented under transient loading. Incorporation of an armour layer and further tests need now to establish that crack propagation can successfully be altered and stopped to produce a material and mock-up with advanced lifetime estimates even in a pre-cracked state. After qualifying the materials the final step will be the actual design of a mock-up incorporating both $W_{f} / C u$ as well as $W_{f} / W$ into a working design that both allows proper heat exhaust as well as resilience against cracking and material damage due to the extreme conditions in the fusion environment. If ready in time an incorporation into a second ITER divertor as a final qualification test could be envisioned. The step to a working design is currently based on a phenomenological approach which in the future has to be supplemented by a design process driven by the requirements from fusion devices, e.g power exhaust, thermal stresses under loading as well as component size.

\section{Acknowledgement}

This work has been carried out within the framework of the EUROfusion Consortium and has received funding from the Euratom research and training programme 2014-2018 under grant agreement No 633053. The views and opinions expressed herein do not necessarily reflect those of the European Commission.

[1] Zinkle, S. J. Physica Scripta, 2016 (2016), T167, 014004.

[2] You, J. et al. Nuclear Materials and Energy, 16 (2018), 1-11.

[3] Ueda, Y. et al. Nuclear Fusion, 57 (2017), 9, 092006.

[4] Ueda, Y. et al. Fusion engineering and design, 89 (2014), 7, 901-906.

[5] Pitts, R. et al. Journal of Nuclear Materials, 438 (2013), S48.

[6] Nygren, R. E. et al. Fusion Engineering and Design, 136 (2018), 10071011.

[7] Muller, A. et al. Fusion Engineering and Design, 124 (2017), 455-459.

[8] Linsmeier, C. et al. Nuclear Fusion, (2017).

[9] Linsmeier, C. et al. Nuclear Fusion, 57 (2017), 9, 092007.

[10] Li, J. et al. Physica Scripta, T159 (2014), 014001.

[11] Philipps, V. Journal of Nuclear Materials, 415 (2011), 1, 2-9.

[12] Coenen, J. et al. Physica Scripta, 2016 (2016), T167, 014002.

[13] Bachmann, C. et al. Fusion Engineering and Design, $98-99$ (2015), 1423-1426.

[14] Coenen, J. et al. Fusion Engineering and Design, 124 (2017), 964-968.

[15] Launey, M. and Ritchie, R. b. Advanced Materials, 21 (2009), 20, $2103-$ 2110 . 
[16] Czel, G. and Wisnom, M. Composites Part A: Applied Science and Manufacturing, 52 (2013), 0, 23-30.

[17] Shimoda, K. et al. Composites Science and Technology, 68 (2008), 1, 98-105.

[18] Koch, F. and Bolt, H. Physica Scripta T, T128 (2007), 100-105.

[19] García-Rosales, C. et al. Fusion Engineering and Design, 89 (2014), 7-8, 1611-1616.

[20] Neu, R. et al. Fusion Engineering and Design, 109-111 (2016), 1046-1052

[21] Calvo, A. et al. Nuclear Materials and Energy, 9 (2016), 422-429.

[22] Litnovsky, A. et al. Nuclear Fusion, 57 (2017), 6, 066020.

[23] Litnovsky, A. et al. Physica Scripta, T170 (2017), 014012.

[24] Liu, D. et al. Journal of Alloys and Compounds, 765 (2018), 299-312.

[25] Calvo, A. et al. International Journal of Refractory Metals and Hard Materials, 73 (2018), 29-37.

[26] Tan, X. et al. Corrosion Science, (2018)

[27] Klein, F. et al. Metals, 8 (2018), 7, 488.

[28] Zinkle, S. and Ghoniem, N. Fusion Engineering and Design, 51 (2000), $0,55-71$.

[29] Stork, D. et al. Journal of Nuclear Materials, 455 (2014), 1-3, 277-291.

[30] Bolt, H. et al. Journal of Nuclear Materials, 307, Part 1 (2002), 0, 43-52.

[31] Lassner, E. and Schubert, W.-D. Tungsten. Springer US (1999).

[32] Hirai, T. et al. Fusion Engineering and Design, 88 (2013), 9-10, 17981801

[33] Boscary, J. et al. Fusion Engineering and Design, 87 (2012), 7-8, 1453 1456.

[34] Matsukawa, M. et al. Fusion Engineering and Design, 83 (2008), 7-9, 795-803.

[35] Missirlian, M. et al. Fusion Engineering and Design, 89 (2014), 7-8, 1048-1053.

[36] Gietl, H. et al. Journal of Composite Materials, (2018), 002199831877114

[37] Tejado, E. et al. Materials Science and Engineering: A, 712 (2018), 738746.

[38] Riesch, J. et al. Acta Materialia, 61 (2013), 19, 7060-7071.

[39] Riesch, J. et al. Physica Scripta, 2014 (2014), T159, 014031.

[40] Jasper, B. et al. Nuclear Materials and Energy, 9 (2016), 416-421.

[41] Jasper et al, B. Material Science Forum, Trans Tech Publications, 825826 (2015), 125-133.

[42] Riesch, J. et al. Nuclear Materials and Energy, 9 (2016), 75-83.

[43] Riesch, J. et al. Physica Scripta, T167 (2016), T167, 014006

[44] Gietl, H. et al. Fusion Engineering and Design, 124 (2017), 396-400.

[45] Mao, Y. et al. Physica Scripta, T170 (2017), 014005.

[46] Mao, Y. et al. Composites Part A: Applied Science and Manufacturing, (2018).

[47] Mao, Y. et al. Nuclear Materials and Energy, 10 (2017), 1-8.

[48] Coenen, J. et al. Nuclear Materials and Energy, (2018).

[49] Müller, A. et al. Nuclear Materials and Energy, 16 (2018), 163-167.

[50] $\mathrm{Hu}, \mathrm{X}$. et al. Journal of Nuclear Materials, (2016).

[51] Duwe, R.; Kühnlein, W. and Münstermann, H. In Fusion Technology 1994, pages 355-358. Elsevier (1995).

[52] Kreter, A. et al. Fusion Science and Technology, 68 (2015), 1, 8-14.

[53] Unterberg, B. et al. Fusion Engineering and Design, 86 (2011), 9-11, 1797-1800.

[54] Yu, J. H. et al. Physica Scripta, 2016 (2016), T167, 014033.

[55] Coenen, J. W. et al. Nuclear Materials and Energy, 12 (2017), 307-312.

[56] Wegener, T. . et al. Nuclear Materials and Energy, 9 (2016), 394-398. ICFRM Conference 2015

[57] Wegener et al, T. In PFMC-2015 (2015). Poster.

[58] Rieth, M. et al. Journal of Nuclear Materials, 442 (2013), 1-3 SUPPL.1, $173-180$.

[59] Koch, F. et al. Physica Scripta, 2011 (2011), T145, 014019.

[60] Causey, R.; Karnesky, R. and Marchi, C. S. In Konings, R. J., editor, Comprehensive Nuclear Materials, pages 511-549. Elsevier, Oxford (2012). ISBN 978-0-08-056033-5.

[61] Maviglia, F. et al. Fusion Engineering and Design, (2018).

[62] Greuner, H. et al. Journal of Nuclear Materials, 367-370, Part B (2007), 1444-1448. Proceedings of the Twelfth International Conference on Fusion Reactor Materials (ICFRM-12)Proceedings of the Twelfth International Conference on Fusion Reactor Materials (ICFRM-12).

[63] Terra, A. et al. Nuclear Materials and Energy, 19 (2019), 7-12. 